\title{
Surveillance to Manage Disease on Canadian Swine Farms
}

\author{
John Berezowski*1, Chris Byra ${ }^{2}$, Egan Brockhoff ${ }^{3}$, Dan Hurnik ${ }^{4}$, Christian Klopfenstein ${ }^{5}$, \\ Harold Kloeze ${ }^{6}$, Luc Bergeron ${ }^{7}$, George Charbonneau ${ }^{8}$, Francois Cardinal ${ }^{9}$, Iqbal Jamal ${ }^{10}$ \\ and Travis Herntier ${ }^{11}$
}

${ }^{1}$ University of Bern, Bern, Switzerland; ${ }^{2}$ Greenbelt Swine Veterinary Services, Chilliwack, BC, Canada; ${ }^{3}$ Prairie Swine Health Services, Red Deer, AB, Canada; ${ }^{4}$ Atlantic Veterinary College, Charlottetown, PE, Canada; ${ }^{5}$ Le Centre de développement du porc du Québec, Quebec, QC, Canada; ${ }^{6}$ Canadian Food Inspection Agency, Owen SOund, ON, Canada; ${ }^{7}$ Ministère de l'Agriculture, des Pêcheries et de l'Alimentation du Québec, Québec, QC, Canada; ${ }^{8}$ South Western Ontario Veterinary Services, Stratford, ON, Canada; ${ }^{9}$ Consultants Avi-Porc, Drummondville, QC, Canada; ${ }^{10} \mathrm{AQL}$ Management Consulting, Edmonton, AB, Canada; ${ }^{11} \mathrm{FD}$ Solutions, Winnipeg, MB, Canada

\section{Objective}

To improve swine farmers and veterinarians ability to manage disease

\section{Introduction}

On a day to day basis, farmers and their veterinarians deal with many diseases without the benefit of surveillance for early outbreak detection, or coordinated outbreak responses. Without this support, highly contagious pathogens such as Porcine Epidemic Diarrhea Virus (PEDv) can spread quickly and potentially cause significant harm. The purpose of this project was to develop a surveillance system to help Canadian swine farmers and veterinarians to deal more effectively with diseases

\section{Methods}

The project team developed some unique approaches to animal disease surveillance. They adopted a unique organizational structure in which the data and information providers (veterinarians and farmers) were also directly involved in the decision making process. They designed an infrastructure that integrated two components 1) a social network and 2) a data collection, analysis, event detection and reporting system

The social network or Swine Veterinary Network (SVN) includes veterinarians and swine specialists. The SVN consists of 3 regional: 1) the Western provinces, 2) Ontario plus the Maritimes and 3) Quebec; and one national network. Each network meets quarterly via the internet. One week prior to the meetings each veterinarian participating in the CSHIN is asked to fill out a web survey where they identify important, changing or emerging disease issues seen in their practice. The data is collated and presented at the meetings. The networks discuss important health issues; develop strategies for dealing with diseases and communicate this intelligence across the country.

The second component is a Practice Based Surveillance (PBS) network. It collects, analyses and reports syndromic data from veterinary practitioners in real time via the internet for early disease detection and to provide other health related information. Because swine farms are relatively large populations in themselves participating veterinarians report farm level data such as the prevalence of syndromes (coughing, diarrhea, neurological etc) within production groups (sows, boars, piglets, etc), as well as individual clinical and laboratory diagnosis

Both networks produce swine health intelligence that is delivered directly to swine farms by participating veterinarians and to other stakeholders through various communication networks. A governance model was developed that allows for control of data by data providers and regional and national decision making processes that include farmers, veterinarians, as well as industry and government representatives.

\section{Results}

Communication was greatly enhance by quarterly meetings and through quarterly regional and national disease updates. The CSHIN was the only national source for PEDv intelligence during the outbreak in Canada, providing daily electronic updates to industry subscribers across the country

The PBS detected a sudden regional increase in swine influenza 3 weeks before it was identified by laboratory submissions. Streptcoccus suis a zoonotic pathogen has been identified as the most common clinical diagnosis on pig farms, providing valuable information to advocate for resources for disease control. Porcine Circovirus 2 was shown to be occurring on swine farms in spite of vaccine use, with improper vaccination being identified as the cause

\section{Conclusions}

The CSHIN infrastructure has been developed and the program rolled out across Canada. It has demonstrated that it can provide value to individual famers and the industry as a whole. Next steps are to secure long term funding

\section{Keywords}

Animal health surveillance; Syndromic surveillance; Social network; Alternative data source

\section{Acknowledgments}

The CSHIN was developed by the Canadian Swine Health Board with funding from Agriculture and Agri-food Canada
*John Berezowski
E-mail: john.berezowski@gmail.com 